\title{
ON THE LOCAL LANGLANDS CONJECTURE IN PRIME DIMENSION
}

\author{
BY PHILIP KUTZKO ${ }^{1}$ AND ALLEN MOY
}

Let $F$ be a local field of residual characteristics $p$. Then it is a conjecture of Langlands [JL] that there should be a natural bijection between the set of $n$-dimensional semisimple representations of the absolute Weil-Deligne group of $F$ and the set of irreducible admissible representations of $\mathrm{GL}_{n}(F)$. Some cases of this conjecture have been established [He, JL, JPS, K, M]. Here we announce further progress toward its verification.

To describe our results, we first note that by work of Bernstein and Zelevinsky $[\mathbf{Z}]$, one may restrict one's attention to irreducible representations of the Weil-Deligne group on the one hand and irreducible supercuspidal representations of $\mathrm{GL}_{n}(F)$ on the other hand. In this context, the conjecture says there should exist a bijection $\sigma \mapsto \pi(\sigma)$ of the set $A_{n}^{0}(F)$ of equivalence classes of continuous, irreducible $n$-dimensional complex representations of $W_{F}$, the absolute Weil group of $F$, with the set $A^{0}\left(\mathrm{GL}_{n}(F)\right)$ of equivalence classes of admissible irreducible supercuspidal representations of $\mathrm{GL}_{n}(F)$. This bijection should satisfy the following conditions:

(1.01) $\epsilon(\pi(\sigma), \psi)=\epsilon(\sigma, \psi)$ (see [D, GJ] for definitions),

(1.02) $\pi(\sigma) \otimes \chi \circ \operatorname{det}=\pi(\sigma \otimes \chi)$ for all quasi-characters $\chi$ of $F^{x}$,

(1.03) $\omega_{\pi(\sigma)}=\operatorname{det} \sigma$, where $\omega_{\pi(\sigma)}$ is the central character of $\pi(\sigma)$.

We note that if $n=1$, the existence of such a bijection is a restatement of the fundamental theorem of local classified theory [S]; thus when $n \geq 2$, the conjecture under consideration may be thought of as a nonabelian analogue of that theorem.

When $n \geq 2$ the construction of $\pi(\sigma)$ breaks naturally into two steps.

I. Construction of $\pi(\sigma)$ when $\sigma$ is induced from a representation of smaller dimension. This construction is provided when $n=2$ by decomposing the Weil representation of $\mathrm{SL}_{2}(F)$ (see [JL]). When $n=3$, it is obtained by global methods [JPS]. When $p \nmid n$ then all $n$-dimensional irreducible representations $\sigma$ of $W_{F}$ are monomial, and one may use a representation which induces $\sigma$ to construct a supercuspidal representation $\pi^{\prime}(\sigma)$ of $\mathrm{GL}_{n}(F)$. This was first done by Howe [Ho], who conjectured that $\pi^{\prime}(\sigma)$ satisfied (1.01)-(1.03). Recently, Moy $[\mathbf{M}]$ showed that a representation $\pi(\sigma)$ satisfying (1.01)-(1.03) may be obtained by a slight modification of Howe's construction and thus verified the Langlands conjecture in case $p \nmid \chi n$ (one needs, however, that char $F=0$ in order that the map $\sigma \mapsto \pi(\sigma)$ be bijective).

When $p \mid n$, however, the above approach appears to fail.

Received by the editors June 27, 1983.

1980 Mathematics Subject Classification. Primary 12B25, 20G05; Secondary 22E50, 12 B15.

${ }^{1}$ Supported in part by NSF Grant \#MP575 07481. 
II. Extension of the map $\sigma \rightarrow \pi(\sigma)$ to primitive representations $\sigma$. Such an extension was obtained in case $n=p=2$ by Kutzko in $[\mathbf{K}]$ and in case $n=p=3$ by Henniart in [He], thus verifying the Langlands conjecture in these cases.

In general, one has reason to expect that step I above may be approached using global methods and that, in particular, such methods will lead to the construction of $\pi(\sigma)$ when $\sigma$ is induced from a one-dimensional representation on a normal subgroup of $W_{F}$. We here announce that, given such a global construction for monomial representations $\sigma$ of dimension $n=p$, we are able to construct $\pi(\sigma)$ for an arbitrary $p$-dimensional representation $\sigma$. To be precise, we must make the following

Assumption 1.1. Let $K$ be a local field of residual characteristic $p$ and let $E / K$ be a cyclic extension of degree $p$. Let $\theta$ be a quasi-character of $E^{x}$ which is not fixed by the galois group, $\Gamma_{E / K}$, of $E / K$ and let $\sigma=\operatorname{Ind}_{W_{E} \uparrow W_{K}} \theta$. Then there is an irreducible supercuspidal representation $\pi(\sigma)$ of $\mathrm{GL}_{p}(K)$ which satisfies (1.01)-(1.03). Furthermore, the map $\sigma \rightarrow \pi(\sigma)$ is injective, and if $L$ is a field over which $K$ is galois, then the map $\sigma \rightarrow \pi(\sigma)$ commutes with $\Gamma_{K / L}$.

We then prove

TheOREM 1.2. Suppose Assumption 1.1 holds for all extensions $K / F$. Then, given any irreducible $p$-dimensional representation $\sigma$ of $W_{F}$, there exists an irreducible supercuspidal representation $\pi(\sigma)$ of $\mathrm{GL}_{p}(F)$ satisfying $(1.01)$ $-(1.03)$.

We proceed as follows (proofs will be provided elsewhere).

Definition 1.3. Call a galois extension $K / F$ a good extension of $F$, either if $\Gamma_{K / F}$ is cyclic and either $[K: F] \mid p-1$ or $[K: F] \mid p+1$, or if $\Gamma_{K / F}$ is a generalized quaternion group and $[K: F] \mid 2(p+1)$.

Proposition 1.4. Let $K / F$ be a good extension. Then there is a map lift $_{K / F}$ which maps irreducible supercuspidal representations of $\mathrm{GL}_{p}(F)$ to irreducible supercuspidal representations of $\mathrm{GL}_{p}(K)$ and has the following properties:

(1.4.1) If $\pi$ is an irreducible supercuspidal representation of $F$, then $\operatorname{lift}_{K / F} \pi$ is fixed under $\Gamma_{K / F}$;

(1.4.2) $\epsilon\left(\operatorname{lift}_{K / F} \pi, \psi \circ \operatorname{tr}_{K / F}\right)=[\epsilon(\pi, \psi)]^{[K: F]} \lambda_{K / F}^{-p} \cdot \delta(\pi)$, where $\lambda_{K / F}$ is the Langlands constant associated to $K / F$ and $\delta(\pi)= \pm 1$; $F^{x}$

(1.4.3) $\operatorname{lift}_{K / F}(\pi \otimes \chi)=\operatorname{lift}_{K / F}(\pi) \otimes \chi \circ N_{K / F}$ for any quasi-character $\chi$ of

(1.4.4) Let $\pi_{K}$ be an irreducible supercuspidal representatin of $K$ which is fixed under $\Gamma_{K / F}$ and let $\omega\left(\pi_{K}\right)$ be its central character. Then if $\omega$ is a quasicharacter of $F$ for which $\omega_{K}=\omega \circ N_{K / F}$, there is exactly one irreducible supercuspidal representation $\pi$ of $\mathrm{GL}_{p}(F)$ for which $\omega(\pi)=\omega$ and $\operatorname{lift}_{K / F} \pi=$ $\pi_{K}$.

Proposition 1.5. Let $\sigma$ be an irreducible p-dimensional representation of $\Gamma_{F}$. Then there is a good extension $K / F$, a cyclic extension $E / F$ and $a$ quasi-character $\theta$ of $E^{x}$ such that $\left.\sigma\right|_{W_{K}}=\operatorname{Ind}_{W_{E} \uparrow W_{K}} \theta$. Furthermore,

$$
\epsilon\left(\left.\sigma\right|_{W_{K}}, \psi_{K / F}\right)=[\epsilon(\sigma, \psi)]^{[K: F]} \lambda_{K / F}^{-p} \delta(\sigma), \quad \text { where } \delta(\sigma)= \pm 1
$$


Proposition 1.6. With notation as above, and assuming 1.1, define the representation $\pi(\sigma)$ of $\mathrm{GL}_{p}(F)$ by the conditions lift $_{K / F} \pi(\sigma)=\pi\left(\left.\sigma\right|_{W_{K}}\right), \omega(\pi(\sigma))=$ $\operatorname{det} \sigma$. Then $\epsilon(\pi(\sigma), \psi)=\xi \epsilon(\sigma, \psi)$, where $\xi$ is a pth-power root of unity.

Proposition 1.7. $\pi(\sigma)$ satisfies conditions (1.01)-(1.03). If the map $\sigma \mapsto$ $\pi(\sigma)$ given by Assumption 1.1 has the additional property that it commutes with lift $K / F$, then the map $\sigma \mapsto \pi(\sigma)$ constructed above is unique and injective. If all irreducible supercuspidal representations of $\mathrm{GL}_{p}(F)$ may be constructed by induction from open compact-modulo center subgroups (see $[\mathbf{C}]$ ), then the map $\sigma \mapsto \pi(\sigma)$ is a bijection of the set of equivalence classes of irreducible $p$-dimensional representations of $W(F)$ with the set of equivalence classes of irreducible supercuspidal representations of $\mathrm{GL}_{p}(F)$ (see also [Ko]).

\section{REFERENCES}

[C] H. Carayol, Représentations cuspidales du groupe linéaire, preprint, Univ. of Paris VII, 1982.

[D] P. Deligne, Les constantes des equations fonctionelles des fonctions $L$, Lecture Notes in Math., vol. 349, Springer-Verlag, 1973, pp. 501-597.

[GJ] R. Godement and H. Jacquet, Zeta functions of simple algebras, Lecture Notes in Math., vol. 260, Springer-Verlag, 1972.

[He] G. Henniart, La conjecture de Langlands locale pour GL(3), Inst. Hautes Étude Sci. notes, 1982.

[Ho] R. Howe, Tamely ramified supercuspidal representations of $\mathrm{GL}_{n}(F)$, Pacific J. Math. 73 (1977), 437-460.

[JL] H. Jacquet and R. P. Langlands, Automorphic forms on GL(2), Lecture Notes in Math., vol. 114, Springer-Verlag, 1970.

[JPS] H. Jacquet, I. Piatetski-Shapiro and J. A. Shalika, Automorphic forms on GL(3), Ann. of Math. (2) 109 (1979), 169-258.

[K] P. Kutzko, The Langlands conjecture for $\mathrm{GL}_{2}$ of a local field, Ann. of Math. (2) 112 (1980), 381-412.

[Ko] H. Koch, On the local Langlands conjecture for central division algebras of index $p$, Invent. Math. 62 (1980), 243-268.

[M] Allen Moy, Local constants and the tame Langlands correspondence, Thesis, Univ. of Chicago, 1982.

[S] J. P. Serre, Corps Locaux, Hermann, Paris, 1968.

[Z] A. V. Zelevinsky, Induced representations of reductive p-adic groups, Ann. Sci. École Norm. Sup. 13 (1980), 165-210.

Mathematical Sciences Division, University of Iowa, Iowa City, Iowa 52242

Department of Mathematics, Yale University, New Haven, Connecticut 06457 
